\title{
PROPOSTA DE ENSINO PARA MÉTODOS DE SEPARAÇÃO DE MISTURAS BASEADA NA RESOLUÇÃO DE PROBLEMAS E SUAS RELAÇÕES COM OS ESTILOS DE APRENDIZAGEM DOS ALUNOS
}

\author{
TEACHING PROPOSAL FOR SEPARATION OF MIXTURES \\ METHODS BASED ON PROBLEM SOLVING AND ITS \\ RELATIONSHIPWITHTHE LEARNING STYLES OF STUDENTS
}

\author{
Cleber Pinto da Silva* \\ Tiago Jacumasso* \\ Sandro Xavier de Campos ${ }^{* *}$
}

\begin{abstract}
RESUMO
O ensino de Química enfrenta algumas dificuldades em relação à aprendizagem de conceitos teóricos. Para tentar reverter essa situação, foi aplicada e analisada uma proposta de ensino baseada na metodologia de resolução de problemas conceituais envolvendo separações de misturas. A proposta foi realizada com 25 alunos do Ensino Médio de uma escola pública da cidade de Ponta Grossa. Os conteúdos foram aplicados e, posteriormente, realizou-se uma análise por meio de questões abertas e fechadas tendo como referência o método psicométrico proposto por Felder e Silverman (1988) para estilos de aprendizagem. Verificouse que a proposta atende os requisitos básicos exigidos pelo MEC, pois trabalha com estilos de aprendizagem variados, o que não ocorre pelas propostas didáticas tradicionais.
\end{abstract}

Palavras-chave: Separação de mistura. Estilos de aprendizagem. Resolução de problemas.

\begin{abstract}
Chemistry teaching faces some difficulties in relation to the learning of theoretical concepts. To try to reverse this situation a teaching proposal, based on a methodology for solving conceptual problems involving separations of mixtures was applied and analyzed. The proposal was carried out with 25 high school students at a public school in Ponta Grossa. The contents were applied and subsequently analysis was performed by means of open and closed questions with reference to the psychometric method proposed by Felder and Silverman (1988) regarding learning styles. It was found that the proposal meets the basic requirements demanded by the Ministry of Education (MEC), since it works with varied learning styles, which does not occur in traditional didactic proposals.
\end{abstract}

Keywords: separation of mixture, learning styles, problem solving.

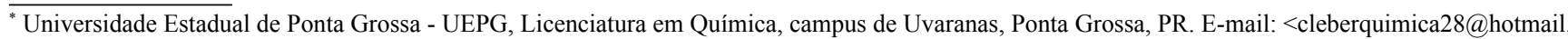
com>,<tiagojacumasso@gmail.com>.

${ }^{* *}$ Universidade Estadual de Ponta Grossa UEPG, Departamento de métodos e técnicas de ensino, Campus Central - Bloco B - sala113 Praça Santos Andrade s/nº Centro, CEP: 84010-790, Ponta Grossa, PR. E-mail: <campos@uepg.br>.
} 


\section{Introdução}

$\mathrm{Na}$ atual estrutura educacional brasileira, um dos maiores problemas enfrentados para o aprendizado dos conceitos de química se refere a estratégias de ensino utilizadas nas escolas por professores do Ensino Básico e Médio.

Muitas vezes, essas estratégias estão focadas na passividade dos alunos, os quais, na maior parte do tempo, participam das aulas apenas como "repetidores" do que o professor fala e escreve e não encontram relações no que aprendem e vivenciam. Assim, o ensino perde o caráter de formação do cidadão, pois o aluno não encontra motivos para ir à escola e se alfabetizar.

Um professor com características da educação bancária não vê na sua pratica pedagógica oportunidades para combater a desigualdade e descriminação social que muitos de seus alunos vivenciam (FREIRE, 1987).

Muitas propostas estão sendo desenvolvidas no sentido de diminuir tais problemas. Uma delas é a metodologia de resolução de problemas, a qual, segundo Carvalho Jr. (CARVALHO JUNIOR, 2002), consiste na criação de grupos tutoriais que devem seguir os seguintes passos: identificação e esclarecimento de termos desconhecidos; identificação dos problemas propostos; formulação de hipóteses; resumo das hipóteses; formulação dos objetivos; estudo individual dos assuntos levantados nos objetivos e retorno ao grupo tutorial para discussão frente a novos conhecimentos adquiridos na fase anterior.

Em suas últimas revisões, o Exame Nacional do Ensino Médio (ENEM) passou a adotar uma pedagogia que possibilita identificar em que área do conhecimento ou competência o aluno está mais ou menos apto, e apontar quais são as suas carências. Segundo o site oficial do Ministério da Educação, "o teste avalia a bagagem cultural do estudante, em vez de analisar, pontualmente, um conteúdo específico". Dessa forma, o ENEM propõe avaliar modalidades de ensino com foco em concepções construtivistas.

Felder e Silverman (1988) verificando um alto índice de reprovação nas matérias com que trabalhavam, elaboraram um método para verificar os estilos de aprendizagem, e, assim, diagnosticar quais eram suas dificuldades. Esse método ficou conhecido como Index of Learning Styles (ILS), e divide os estilos em quatro categorias: ativo-reflexivo; sensorial-intuitivo; visual-verbal e sequencial-global, sendo que todos possuem peculiaridades entre si.

Gardner (2001) afirma que cada indivíduo possui uma mistura diferenciada de inteligências ou inteligências múltiplas. Dessa forma, cada ser humano tem um processo cognitivo particular, o que individualiza a forma de tudo que aprendemos, por meio de vários processos que influenciam a formação de um indivíduo, seja de cunho social, cultural e/ou econômico.

Desta forma, o presente trabalho tem como objetivo o desenvolvimento e avaliação de uma estratégia didática utilizando PBL para o ensino de conceitos de separação de misturas.

\section{Materiais e Métodos}

Os grupos tutoriais, trabalhos de pesquisa, resposta aos questionários e discussões foram realizados com vinte e cinco alunos do primeiro ano do Ensino Médio de uma escola pública de Ponta Grossa/PR em três horas aula.

A primeira aula foi utilizada para a introdução do problema: com uma carta fictícia encaminhada pela companhia de saneamento do Paraná (SANEPAR) à Universidade Estadual de Ponta Grossa (UEPG), solicitou-se uma análise do material que causou o rompimento da adutora do rio Pitangui devido a um entupimento na tubulação, bem como um método para separar esse material. Em seguida, foram exibidos fotos e vídeos da adutora para melhor entendimento dos alunos.

Aplicou-se um questionário com duas perguntas específicas sobre o tema: 1) "O que você sabe sobre separações de misturas?”; 2) "Quantos métodos de separações de misturas são utilizados em sua casa, diariamente? Cite, no mínimo, três exemplos".

Posteriormente, formaram-se grupos de quatro alunos, os quais deveriam propor objetivos e formular hipóteses para o que estaria ocorrendo e qual seria a possível solução para o problema.

Em seguida, os grupos se reuniram e discutiram todas as informações e conceitos adquiridos nas pesquisas. Em outro momento, cada grupo elaborou uma proposta para resolver o problema tendo que indicar, de forma oral, suas ideias para solucionálos, que foram escritas no quadro negro pelos tutores 
(pesquisadores), com a intenção de registrá-las para dar sequência à discussão.

Os grupos foram convidados a eliminar as propostas que não correspondiam à resolução dos problemas e, assim, passaram a discutir quais seriam as mais coerentes até chegar a um consenso.

Utilizou-se, posteriormente, uma aula para a aplicação do questionário de estilos de aprendizagem. Para a análise das repostas houve uma divisão em quatro grandes dimensões de estilos de aprendizagem: ativo e reflexivo; detalhista e generalista; visual e verbal; sequencial e global. Cada dimensão possuía onze questões, cada uma com duas alternativas, que, dessa forma, somavam vinte e duas possibilidades. O resultado determinou a qual dimensão o aluno tem mais afinidade (LITZINGER, LEE, WISE, FELDER, 2007).

\section{Resultados e discussão}

A tabela 1 apresenta os resultados sobre os conhecimentos prévios dos alunos em relação ao nível de entendimento sobre conceitos relacionados a separações de misturas.

Tabela 1 - Categorização das respostas à pergunta: "O que você sabe sobre separações de misturas?”.

\begin{tabular}{l|l}
\hline Estilos de respostas & Total de alunos \\
\hline Completa & 14 \\
\hline Parcial & 3 \\
\hline Sem sentido & 8 \\
\hline
\end{tabular}

As respostas que foram consideradas completas para essa questão continham parte da compreensão dos conceitos científicos envolvidos. Como exemplo, cita-se a resposta do aluno A: "é o método de separar substâncias diferentes como sólido líquido, por exemplo, água e areia”. As respostas parciais somam $12 \%$. O que foi observado nessa categoria foram conceitos dispersos, que têm fundamento, mas que por si só não explicam todo o processo de separação de misturas, deixando falhas na explicação. Foram categorizadas como sem sentido as respostas que não têm relação ao que se pergunta. Um exemplo é a resposta do aluno C: "sabemos que cada processo tem sua função e outros ajudam entender mais o que cada experimento químico realiza", somando $32 \%$ das respostas.
Podemos observar nas respostas que muitos alunos, mesmo sem nunca terem aulas a respeito do conteúdo, já possuíam conhecimento do que eram separações de misturas. Esse conhecimento prévio é importantíssimo para que haja a compreensão sobre o processo de separação de mistura. $\mathrm{O}$ aluno começa a participar das aulas de um modo natural e espontâneo, visualizando acontecimentos do seu dia a dia, descartando aquela ideia de que a disciplina de Química é uma matéria difícil de aprender (CHASSOT, 2011).

A tabela 2 apresenta os resultados das propostas individuais que foram elaboradas após a pesquisa sobre o problema proposto.

Tabela 2 - Resultados das respostas para o problema proposto. "Como evitar o rompimento da adutora da Sanepar do rio Pitangui, por acúmulo de materiais que estão entrando e acumulando na tubulação".

\begin{tabular}{l|c|l}
\hline Resultados & $\begin{array}{l}\text { Total de } \\
\text { alunos }\end{array}$ & Estilos de respostas \\
\hline Soluções corretas & 19 & Solução para o problema proposto \\
\hline $\begin{array}{l}\text { Conscientização } \\
\text { Ambiental }\end{array}$ & 5 & $\begin{array}{l}\text { Propostas que solucionariam o } \\
\text { problema, mas demandariam um } \\
\text { longo tempo e não utilizariam } \\
\text { métodos de separação. }\end{array}$ \\
\hline Soluções erradas & 1 & $\begin{array}{l}\text { Foram elencadas nessa categoria } \\
\text { as respostas que não apresentaram } \\
\text { uma solução para o problema. }\end{array}$ \\
\hline
\end{tabular}

As soluções que foram apontadas como corretas têm como principal método de separação a filtração e descrevem um processo real que seria eficaz na solução do problema, como a resposta do aluno D: "Colocar uma peneira na entrada da adutora e um filtro logo depois".

Outras propostas estão relacionadas às mudanças de hábitos e podem contribuir para resolver o problema, entretanto não possuem relação com o tema, como a resposta do aluno E: "Pode ser feito a conscientização das pessoas para pararem de poluir, e a limpeza dos tubos". Observou-se no decorrer das análises a construção gradativa do conhecimento, pois nas primeiras perguntas havia muitas respostas baseadas apenas no senso comum. A partir das pesquisas propostas pelos alunos, notouse uma crescente aquisição de conhecimentos. Segundo Dewey (2003) é necessário que se pense na educação como uma reconstrução contínua de experiências. 
Após as análises dos materiais coletados sobre a metodologia de resolução de problemas forma aplicados questionários elaborados seguindo o modelo proposto por Felder (2007) para determinar os estilos de aprendizagem dos alunos envolvidos nesse trabalho.

A tabela 3 representa o índice de estilos referentes à dimensão ativo $\mathrm{X}$ reflexivo.

Tabela 3 - Resultados obtidos por meio do questionário e análise da dimensão ativo / reflexivo.

\begin{tabular}{l|l|l|l|l|l|l|l|l|l|l|l|l}
\hline & \multicolumn{5}{|c|}{ Ativo } & \multicolumn{7}{c}{ Reflexivo } \\
\hline Pontuação & 11 & 9 & 7 & 5 & 3 & 1 & 1 & 3 & 5 & 7 & 9 & 11 \\
\hline Alunos & & & $\mathbf{2}$ & $\mathbf{5}$ & $\mathbf{1}$ & $\mathbf{5}$ & $\mathbf{6}$ & $\mathbf{4}$ & $\mathbf{2}$ & & & \\
\hline
\end{tabular}

Observou-se que cinco alunos têm afinidade moderada com o estilo ativo, pois obtiveram a pontuação cinco, o que indica que podem ter algum déficit de aprendizagem, caso o professor trabalhe somente com explicações e deixe de lado as aplicações dos conceitos.

Apenas dois alunos têm pontuação cinco e tiveram uma relação moderada com o estilo reflexivo. Esses alunos podem ter problemas com estudos ativos, caso o professor trabalhe somente com aplicações do conteúdo na forma prática. O restante dos alunos apresentou relações fracas em ambos os etilos dessa dimensão, de modo que conseguem se adaptar com mais facilidade aos dois estilos.

A tabela 4 representa o índice de estilos referentes à dimensão detalhista/sensorial X generalista/ intuitivo.

Tabela 4 - Resultados obtidos por meio do questionário e análise da dimensão detalhista /generalista.

\begin{tabular}{l|c|c|c|c|c|c|c|c|c|c|c|c}
\hline & \multicolumn{4}{|c|}{ Detalhista /sensorial } & \multicolumn{5}{c}{ Generalista/intuitivo } \\
\hline Pontuação & 11 & 9 & 7 & 5 & 3 & 1 & 1 & 3 & 5 & 7 & 9 & 11 \\
\hline Alunos & & $\mathbf{2}$ & $\mathbf{5}$ & $\mathbf{3}$ & $\mathbf{5}$ & $\mathbf{3}$ & $\mathbf{1}$ & $\mathbf{3}$ & $\mathbf{3}$ & & & \\
\hline
\end{tabular}

Nessa dimensão dois alunos alcançaram a pontuação nove para o estilo detalhista / sensorial, o que indica que podem ter uma dificuldade mais elevada no trabalho com informações que carecem de memorização e exigem inovação.

Oito alunos obtiveram pontuação entre cinco e sete e podem ter dificuldades moderadas em trabalhar com conteúdos que exijam intuição.
No estilo generalista/intuitivo três alunos podem apresentar dificuldades moderadas em trabalhar as informações detalhadamente, pois possuem uma pontuação cinco e tendem a absorver os conteúdos de forma mais geral, sem muitos detalhes. O restante dos alunos apresentaram pontuação entre um e três e podem ter facilidade em trabalhar em ambos os etilos.

A tabela 5 representa o índice de estilos referentes à dimensão visual $\mathrm{X}$ verbal.

Tabela 5 - Resultados obtidos por meio do questionário e análise da dimensão visual $\mathrm{X}$ verbal.

\begin{tabular}{l|c|c|c|c|c|c|c|c|c|c|c|c}
\hline & \multicolumn{7}{|c|}{ Visual } & \multicolumn{7}{c}{ Verbal } \\
\hline Pontuação & 11 & 9 & 7 & 5 & 3 & 1 & 1 & 3 & 5 & 7 & 9 & 11 \\
\hline Alunos & & & $\mathbf{1}$ & $\mathbf{5}$ & $\mathbf{7}$ & $\mathbf{1}$ & $\mathbf{5}$ & $\mathbf{3}$ & $\mathbf{2}$ & $\mathbf{1}$ & & \\
\hline
\end{tabular}

Para essa dimensão, seis alunos possuem características de estilos visuais e podem apresentar dificuldades moderadas em relação à leitura e compreensão de materiais escritos. Em relação ao estilo de aprendizagem verbal, três alunos possuem pontuação entre cinco e sete e podem encontrar dificuldades quando o trabalho é feito de forma visual, ou seja, professores que usam muita animação de imagens ou vídeos.

A tabela 6 representa os estilos referentes à dimensão sequencial X global.

Tabela 6 - Resultados obtidos a partir do questionário e análise da dimensão sequencial X global.

\begin{tabular}{l|c|c|c|c|c|c|c|c|c|c|c|c}
\hline & \multicolumn{5}{|c|}{ Sequencial } & \multicolumn{7}{c}{ Global } \\
\hline Pontuação & 11 & 9 & 7 & 5 & 3 & 1 & 1 & 3 & 5 & 7 & 9 & 11 \\
\hline Alunos & & $\mathbf{1}$ & & $\mathbf{4}$ & $\mathbf{5}$ & $\mathbf{6}$ & $\mathbf{3}$ & $\mathbf{4}$ & $\mathbf{1}$ & & $\mathbf{1}$ & \\
\hline
\end{tabular}

Nessa dimensão verificou-se que um aluno possuiu pontuação nove referente ao estilo sequencial, ele pode ter grande dificuldade quando o professor generaliza os conteúdos. Quatro alunos possuem pontuação cinco e têm uma afinidade moderada, de modo que podem encontrar dificuldades em trabalhar de forma mais generalizada.

No estilo global um aluno alcançou a pontuação nove. Quando os conteúdos são trabalhados detalhadamente é possível que tal aluno encontre grande dificuldade, pois perde a concentração facilmente de maneira que pode deixar de lado informações importantes. Um aluno apresentou relação moderada 
com o estilo global podendo ter dificuldades acentuadas em informações transmitidas sequencialmente. O restante dos alunos possui afinidade fraca e dificilmente terá dificuldade em alternar entre os dois estilos.

Nas análises feitas por meio das questões propostas pode-se verificar certa diversidade de estilos de aprendizagem em uma única sala de aula. Em vários casos, a dificuldade observada estava na compreensão dos métodos de separações de misturas e não no conhecimento teórico que envolvia as perguntas e métodos propostos.

Em situações simples que envolviam apenas a lógica alguns alunos não souberam responder porque tentaram elaborar conclusões mais complexas, não conseguiram interpretar as perguntas ou não relacionaram o conteúdo com o seu cotidiano. Segundo Perrenoud (2001), toda pessoa tem valores, hábitos e até mesmo manias, gostos e desgostos, desejos, medos, fragilidades e obsessões, egoísmos e entusiasmos. Dessa forma, o aluno, quando observa sua fragilidade em relação à aprendizagem muitas vezes passa a apresentar comportamentos inadequados para uma sala de aula.

\section{Considerações finais}

A proposta de ensino para separação de misturas por meio da resolução de problemas se mostrou uma alternativa para superar os índices de deficiência sobre os conceitos trabalhados. Acredita-se que isso ocorreu porque a turma avaliada possuía uma diversificação muito grande em relação aos estilos de aprendizagem. Podemos verificar, por meio das respostas, um ganho expressivo em relação ao conhecimento. No primeiro questionário o conhecimento estava fundamentado no senso comum. Após a elaboração da pesquisa por cada aluno e o desenvolvimento da resolução de problemas pelos grupos verificou-se uma evolução conhecimento cientifico. As discussões já não estavam baseadas apenas em como ocorrem às separações, mas sim nos motivos pelos quais elas ocorrem e quais as características químicas e físicas que levam aos processos de separações de misturas.

Por meio das análises das respostas sobre os diferentes estilos de aprendizagem, constatou-se que esse grupo de alunos possui formas diversificadas de aprender conceitos científicos. Assim, acredita-se na necessidade de que seus professores disponibilizem vários canais de aprendizagem por meio de diferentes estratégias de ensino como, por exemplo, a resolução de problemas.

\section{Referências}

CARVALHO JÚNIOR, P. M. Modelo de uso da tecnologia de informação no suporte ao processo de ensinoaprendizagem baseado em problemas no curso médico: desenvolvimento e avaliação. Tese de Doutorado. Campinas, UNICAMP, 2002.

CHASSOT, A. Alfabetização científica: questões e desafios para a educação. Ijuí. Unijuí, 2011. p.101-114.

DEWEY, J. Pedagogos e pedagogías. Revista Educación Hoy Bogotá, D.C. Colômbia Revista de la confederación Católica, 2003. p.98-99.

FELDER, R.M., SILVERMAN, L. K. Learning and teaching styles in engineering education. Engineering Education. v.78, n. 7, 1988.

FREIRE, P. Pedagogia do oprimido. 17ed. Paz e Terra. Rio de Janeiro. 1987. p.37-42.

GARDNER, H. Inteligência: um conceito reformulado. Rio de Janeiro: Objetiva, 2000. p. 46-47.

LITZINGER, T. A; LEE, S. H.; WISE, J. C.; FELDER, R. M. "A psychometric study of the index of learning styles". Journal of Engineering Education, 2007.

PERRENOUD, $P$. A pedagogia nas escolas das diferenças: fragmentos de uma sociologia do fracasso. Porto Alegre: Artmed, 2001. p.24-25.

Disponível em: $<$ http://portal.mec.gov.br/index.php?option $=$ com_content\&task $=v i e w \& i d=2414>$. Acesso em: 09 maio. 2013.

Recebido em 31/01/2013 Aceito em 17/04/2013, 